\title{
Absurdism and Generation Z Humor: The Effects of Absurdist Content on Perceived Humor Levels in Generation Z Students
}

\author{
Chloe Partlow ${ }^{1}$ and Patricia Talarczyk" \\ ${ }^{1}$ Mentor High School, Mentor, OH, USA \\ \#Advisor
}

$\underline{\text { ABSTRACT }}$

This study investigated the relationship between absurd humor in meme stills and the perceived humor among Generation $\mathrm{Z}$ students. Students of a high school in Northeast Ohio were given one of two seemingly identical surveys, each with a selection of six memes and individual Likert scales corresponding to the presented memes. A rubric was created with guidelines for visual components that was used to assign the presented memes an absurdity score with categories of "minimal absurdism," "semi-absurd," and "absurd." Participants (n=298) were asked to rank the memes on the Likert scale ranging from a value of 1 -not at all funny to a maximum value of 5-extremely funny. Following a chi-squared test, we can be over $99 \%$ confident that there is a present relationship between absurdist humor content in meme stills and how humorous they are rated by Generation $\mathrm{Z}$ students. These findings can be applied to usage of memes in marketing, an ever-increasing function of meme humor, to make advertisements and communication more effective.

\section{Introduction}

Humor on the internet today is starkly different from the humor popularized in the late 2000s; rather than thick impact font overlaid on an image, punchline and context obvious, the blurred image of a tearful cat and its stocky limbs with an apple as a body and the text "Bingobingobingo" off-center sends teenagers reeling (Appendix A). A lengthy description of an image certainly, but there are few better ways to describe the still with its amalgam of content-chaos with an overwhelming simplicity. Memes today may require significant context and knowledge of obscure events to understand the joke, and others are downright incomprehensible. Generation $\mathrm{Z}$ may be most engaged with the internet, having spent most of their lives with internet access while sharing copious amounts about their personal lives online (Turner, 2015). With a significant portion of Generation Z living online and conducting their social endeavors through social media, these memes may reflect a shift towards absurdity in humor.

As a philosophical school of thought, absurdism is based fully around the principles of chaos; as is understood away from the philosophical lens, chaos and absurdity are generally considered different names for the same thing. Albert Camus, a prominent twentieth-century Algerian philosopher, may be the most prominent absurdist philosopher for his fictional works The Stranger (L'Étranger) and The Myth of Sisyphus (Le Mythe de Sisyphe), both originally published in 1942; from these works, absurdism was seen to be the understanding that life has no greater purpose or meaning despite the human desire for such greater, universal purpose or meaning (Dotterweich, 2019). Following the understanding that life has no great plan, people are meant to find value in their relationships and experiences rather than the fulfillment of a greater agenda from outside forces (Dotterweich, 2019). Absurdism both as a concept in humor and as a philosophy is certainly present and recognizable in meme stills, from the apple-cat to the genre of memes set on poking fun at reality and the idea that life has a set meaning or purpose. Generation Z, with a disputed 
birth date commonly accepted from the mid-90s to the early 2000s, was born into digital life and much of their generated content online reflects nihilism and existentialism-both ideas parallel to absurdity in the idea that life has no meaning but the individual is a free agent—but tends to remain optimistic with regards to the future of the world and their place in it (Brown, 2020).

The modern meme has found its way into scholarly conversations which analyze a number of components of memes and the relevant internet culture. Memes have been studied from their use as a form of speech all the way to their presence in political discourse. A study by M. Sălcudean and R. Motoroiu-Ştefan analyzes the accessibility of memes as well as how they relate to language and humor. This study also discusses the classification of memes in common Internet terms (Sălcudean \& Motoroiu-Ştefan, 2020). The classification of memes also shows a basis for the classification of the types of humor found in memes. In one study by Jennifer O'Meara, the use of film dialogue as memes and political commentary is analyzed. The repurposing of film dialogue was found to allow the creators of the meme and the audience to engage in lighter discourse where the addressee of the meme is based on context (O'Meara, 2018). In this study, O'Meara notes how the ridicule of political figures, as was often the goal of the meme, connected the audience to the greater political conversation. Memes have been utlized in many political arenas, even allegedly by Russian nationals during the 2016 United States election (Woodworth, 2018). Furthermore, a study by Emily Apter analyzes the virality of memes and how one event may act as a catalyst for memes as a form of political discourse. Memes used as political discourse may stigmatize their target but act as recordings of political events that remain in historical memory (Apter, 2019). These studies on the role of memes in political discourse display practical consequences of meme culture. An emphasis is also placed on the virality of memes and the constant stream of new content that creates a practically endless and ever-changing resource. One study from 2016 done by S. Cannizzaro posits that digital culture can best be analyzed with a precise semiotic approach with regards to content and virality. Even the use of Victorian memes and its influence on Twitter has been studied with how applicable the references are to jokes and culture (Bourrier, 2016). The existing research on memes reflects a strong academic conversation with numerous lenses and applications. With regards to absurdism and humor, a study from A. van der Wal et al. analyzed popular television shows among teenagers and created a framework to codify types of humor and their prevalence in teen media. The presence of absurdity was analyzed in this study among other types of humor, demonstrating the prevalence of absurdity in media popular amongst youth (van der Wal et al., 2020).

In researching memes and becoming acquainted with the body of knowledge, a clear issue becomes apparent. Few studies published in recent years analyze memes popular among younger demographics and have based their findings on types of memes no longer in the mainstream. In a study performed by L. Grundligh, Grundligh found that memes act similarly to a number of speech acts outlined in another study. This outlines a unique effect of memes as a manner of communication that is layered and incredibly specific to its context. While this study is relevant in terms of its analysis of memes and their functions in communication, it presents an issue found to be common in relevant studies. The memes presented and analyzed in a study two years old are memes that are far from relevant in the current digital culture, especially among Generation $\mathrm{Z}$ and related online communities. Even in the study from Sălcudean and Motoroiu-Ştefan, the meme classifications drawn from their research reflect common but limited genres of memes pulled from sites used less by younger demographics. The lack of more modern memes shared by Generation $\mathrm{Z}$ depicts a wholly different sense of what a meme is and how it can communicate outside of memes that state their intentions outright. The analysis of absurdism as it contributes to humor is also lacking. With plentiful sources and analyses of the works of Albert Camus and other major absurdists, the application of absurdist philosophy to current humor is also lacking.

The focus of this study is to understand how absurdism manifests in memes and how this affects their level of humor among Generation Z, with the potential to better understand current internet culture. For the purposes of this study, a meme still will be defined as any image edited and circulated online with perceived humorous intent and members of Generation Z will be defined as anyone born between the years 1996 and 2007, and will be narrowed to current high school students. Analyzing the current state of memes is integral to meaningful analysis of the cultural impact memes have and their role in communication, as well as their usage beyond a typical social exchange. 


\section{Methods}

To understand the relationship between absurdism and the humor of memes, data had to be collected on how humorous members of Generation $\mathrm{Z}$ found select memes with varying levels of absurdist humor. Absurdism is a recognized form of humor prevalent in media with a strong teenage audience (van der Wal et al., 2020). A survey was created through Google Forms to be distributed amongst students at a local public high school in Northeast Ohio. There were two versions of the survey with different selections of six memes each in order to create variability in the images presented to participants and collect a wider range of data without a large time commitment from participants. Consult Appendix $\mathrm{B}$ to see the sample likert scale and view Appendix $\mathrm{C}$ for the memes presented in both surveys. The method was designed to reach the largest possible sample with many grade levels and educational tracks represented so that the results could be analyzed in the context of a more general high school population.

\section{Survey}

The Google Form format was selected to reach a larger sample size as this format is familiar to many participants in the target population and encourages honesty from participants as they can avoid judgment from their peers, making the data more resistant to response bias. Memes were selected from a variety of online sources, including Twitter, Instagram, and Tumblr, among others. The current nature of memes makes them difficult to properly source and attribute as they are passed along and edited constantly, as well as appearing on many different social media platforms. Memes were selected based on content and whether they appeared to be more absurd or simple upon first inspectionsfrom here, they were divided among the two surveys. Although many memes popular with Generation $\mathrm{Z}$ contain profanity or other content inappropriate for a school environment (i.e. jokes about drug use, sexual activity, or mental health), the memes presented to participants were appropriate in a school environment and contained nothing that would be potentially offensive or harmful to participants. This selection minimized risk to participants and ensured the survey could be distributed to minors without ethical concerns raised by mature content. Two surveys were developed to be distributed; both followed the exact same format with the only variable being the memes presented to the participants. Participants were fully unaware as to what version they received, critical to preventing participants from discussing their responses before submission. Participants were also unaware of the two survey nature of this study to limit discussion and any bias that would arise. This two-survey method allowed for further randomization of the results and participants, and could reduce bias caused by discussion amongst participants.

Prior to the review of memes in the survey, participants were asked for only two pieces of demographic information: their grade level and most used social media. These questions were intended to allow for comparisons in humor level and to see if there was any correlation between social media usage or grade level and how funny a participant found the memes. Participants could be grouped by grade level without having to disclose their age or other personal information.

Participants were presented with a meme labeled "Meme \#1-6" followed immediately by a Likert scale before proceeding to the next image. The question posed asked participants how "humorous" they found the preceding meme and collected their response via a Likert scale. The Likert scale ranged from 1, "not at all funny," to 5, "extremely funny." The use of "humorous" in the question was intended to guide participants while avoiding bias, whereas "funny" was used on the Likert scale itself to simplify the question and response process for a younger demographic; while the survey intends to measure humor, the more common "funny" helps participants to better understand what is being asked of them and how to effectively record their responses. Likert scales have been used to quantify humor before for both children and adults; in one particular study, children and adults viewed sarcastic humor and used a Likert scale to record their ratings (Glenwright et al., 2017). In this study, a Likert scale was the best option to compile 
quantitative data and to promote ease of use for participants; Likert scales are simple and allow for a variety of responses without overwhelming participants with free or unguided responses.

\section{Participant Selection}

In selecting participants, the most effective method of obtaining responses from Generation $\mathrm{Z}$ would be to sample high school students. This study sampled students from a public high school in Northeast Ohio with a student body of over 2500 students ranging from ninth through twelfth grade. The graduating classes of this particular high school range from 2021 to 2024, setting the population firmly into Generation $\mathrm{Z}$ with the assumption that students are graduating at eighteen to twenty years old. High school students have the ability to respond to a survey with little direction beyond what is provided in the Google Form itself and are likely to seek breaks from class time which a survey would provide. Sampling from high school students was intended to increase response and create a larger sample while controlling confounding variables such as a greater age range or differences in social media usage between different age groups. Furthermore, any student who uses social media is bound to be exposed to memes; any student within this high school can participate and provide relevant insight and responses to the survey.

\section{Distribution}

After procuring the staff directory from this high school, a list of names and emails of the English teachers was created. At this particular school, students of all grade levels are required to take some form of English course each year. Teachers were selected who taught a variety of classes including the regular, honors, and Advanced Placement courses within the English department. English teachers were provided with the link to one of the two surveys and distributed the survey amongst their students. Doing so allowed for the largest possible sample size and ensured students of all grade levels and course types were represented. Students at this high school are provided with one-on-one technology which they can access both at school and at home; consistent wifi is provided in the school building and much of the student body has wifi access at home, enabling participants to respond without biasing the responses. The content presented in the survey was screened to be school-appropriate so the survey could be taken at school and without the need to procure parental consent for participation. Students were asked for their assent to participate to inform them of the nature of the survey, and it was made clear in the assent that participation was voluntary and anonymous without compensation, as seen in. As this study was performed during the COVID-19 pandemic, distributing this survey remotely was the best option to mitigate the risk and spread of COVID-19 between the participants and researcher. The distribution of the survey was intended to reach the greatest potential sample and to minimize exposure during the COVID-19 pandemic. Procuring responses remotely also controls for the bias the researcher may inadvertently present to participants. Collecting responses remotely, however, may have influenced the participants' perceptions of the memes and their humor scores as memes, typically a social phenomenon, were limited to interaction solely between the participant and the Google Forms.

\section{Results \& Analysis}

The two surveys received 308 responses collectively with 298 participating, meaning that 10 responses did not assent to participate in the study. Of the participants, $168(57.3 \%)$ were freshmen, $25(8.5 \%)$ were sophomores, $99(33.8 \%)$ were juniors, and $1(0.3 \%)$ was a senior. The most frequently reported social media site was TikTok, totalling 106 responses between both surveys; Snapchat followed with 94 responses. TikTok, commonly used for sharing 15 to 60 second videos, describes itself as "the leading destination for short-form mobile video," in its "About" page. Snap Inc., under its "About" web page, describes the app as "empower[ing] people to express themselves, live in the moment... and have fun together." The app is most commonly used to share media such as images and video along with 
chat functions. For more information on the most frequently used social media sites as reported by participants, consult Appendix D. On any of the reported social media sites, both prompted or listed by the respondent in an "Other" option, memes can be shared. These social media sites have the potential to influence what meme formats participants were exposed to regularly and could be further analyzed for meme humor and preferences as they relate to different social media platforms.

For the purposes of this paper, memes from Survey 1 will be referred to as "Meme A1" or "Meme A3," numerically as they appear on the survey; the same system will be used to refer to the memes from Survey 2 , instead referring to them as "Meme B1" and so on. As can be seen in Table 1 and Table 2, the memes were generally ranked as "unfunny." Most memes were weighted more heavily on the less funny side of the Likert scale which could have been contributed to by the more "clinical" presentation of the memes, referring to their presentation not in a typical social situation but instead in the form of a survey. Some did, however, have positive results on "funny" rankings when put through a chi-squared goodness of fit test, suggesting that some of the memes were considered funny by a significant portion of participants (Appendix E). The memes presented, while providing data suitable for analysis, were potentially regarded as unfunny for a number of reasons. The first reason being that these memes were selected to sample a range of absurd content rankings for participant exposure and likely did not reflect the memes participants encountered in their own online circles. Secondly, these memes were screened for inappropriate content, including but not limited to, swearing, drug paraphernalia and references, suicidal ideation, or references to criminal acts, all of which are common in teen internet culture but were deemed inappropriate for participants in a school setting. While these memes were not perfect representations of the content participants encountered, they were close in their accessibility and references to popular Generation Z culture.

Table 1. Survey Version 1 Likert Scale Responses

\begin{tabular}{lllllll}
\hline Likert Scores & & & & & \\
& Meme A1 & Meme A2 & Meme A3 & Meme A4 & Meme A5 & Meme A6 \\
\hline 1 & 125 & 104 & 84 & 87 & 102 & 101 \\
2 & 39 & 51 & 34 & 48 & 49 & 37 \\
3 & 39 & 39 & 44 & 30 & 22 & 39 \\
5 & 20 & 7 & 34 & 12 & 15 & 15
\end{tabular}

Table 2. Survey Version 2 Likert Scale Responses

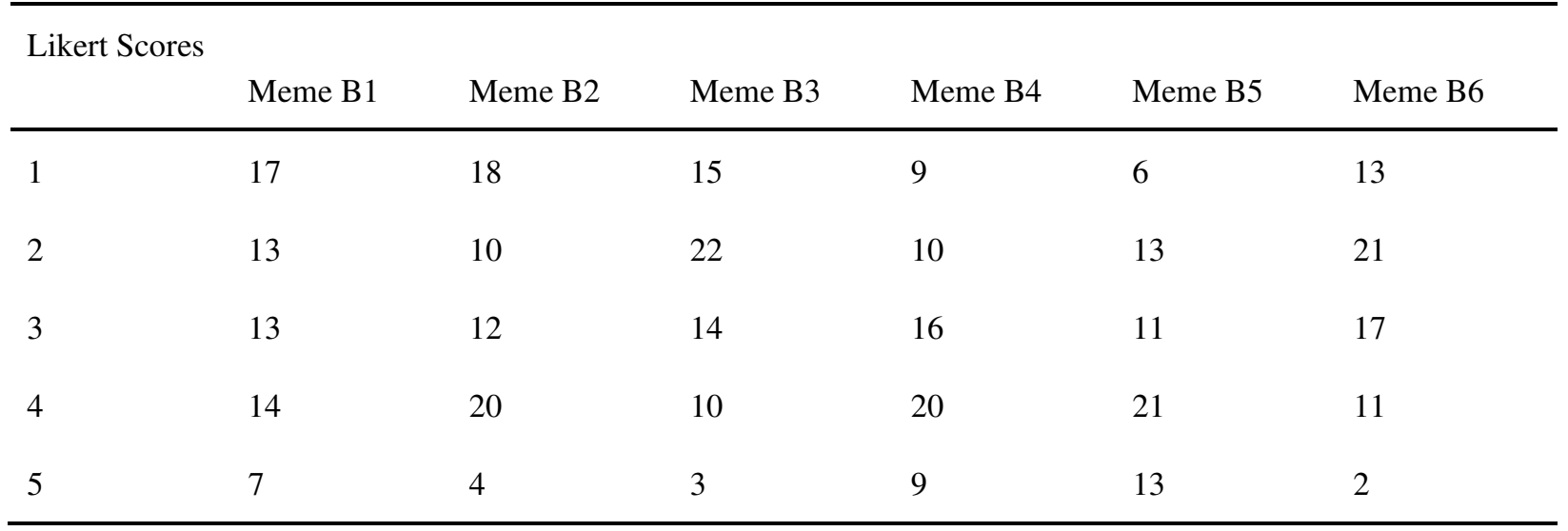


During the data collection process, a rubric was created to analyze the memes selected and give them a definitive score on absurdity content. This rubric can be found in Appendix F. Five factors were defined from analysis of the study done by van der Wal et al. and from observations of the components of the presented memes. Points were awarded to memes that matched the more absurd factors on the rubric. Memes were scored on five factors: the punchline/joke, characters, text, visuals, and context. The punchline factor referred to the overall joke and whether or not it subverted expectations or was well known. The character factor referred to the main character of the visual still, often a human or animal; if the character was well-known or aligned with the joke, the meme was less absurd and vice versa. The text and visual factors considered the visual elements of the still and their harmony with one another, along with how easily read a meme was. "Deep-fried" memes are their own subset of meme culture, referring to manipulated images that are deeply saturated and low quality images. Finally, context was a factor which considered if context was needed to understand the meme and if such was provided; this was weighted higher than the other factors as context can limit the chaotic aspects of absurdism. Memes with an absurdity rating between zero and four were considered "minimal" or minimally absurd. Those with a score of five to eight or nine to 12 were considered "semi-absurd" or "absurd," respectively. The scores of each meme according to the rubric can be found in Appendix G. These scores were used to quantify the absurdity of the memes presented to participants and made the data clearer when comparing Likert scale distributions and the memes' rubric scores.

Figure 1 is the absurdity rubric in full, detailing the criteria for each factor. For meme B1, a score of 10 was awarded. Two points were awarded for the punchline, as the punchline is difficult to find or understand as a joke and could not be easily anticipated. Two points were awarded for the character, being a unique cross of a cat and George Costanza from the American sitcom Seinfeld which is nondescript to the typical viewer and requires knowledge of a specific episode of a facet of popular culture. No points were awarded for text, being easy to read and one font, and two were given for visuals, with low-quality in general and slight variations in quality between the elements of the image. Four points were given for context as the joke has no relevant context to make it more humorous and is not based on a common topic of humor.

Figure 1 Absurdity Factor Rubric

Factors

None (0 points)

Some (1 point)

Significant (2 points)

\section{Punchline/Joke}

How easily anticipated is the punchline or basis of the joke?

\section{Characters}

Are the characters known? Do they make logical sense in context?

\section{Text}

Are the fonts/colors appealing or consistent?
The punchline is expected and logical. pectations or subverts them, but is still somewhat anticipated.

The main character (if app.) is recognizable or requires little context to understand.

One font is used with a single color throughout. Easy to read.

One to two fonts present with more than one color. The font may be challenging or odd.

The main character (if app.) is somewhat unknown or unusually aligned to the joke.
The punchline is unexpected/illogical. The joke is delivered in an odd order or could not be anticipated.

The main character (if app.) is non-descript or unknown/requires significant context to recognize

More than two fonts with several colors. Difficult to read or follow at first glance. 


\section{Visuals}

Is the image quality consistent? Is there harmony in the visual components?
The image quality is consistent The image quality may be low or if not high overall. The visual components make sense together. intentionally manipulated. The components may be questionably related.
The image quality varies from component to component/is manipulated to be low. The components make little logical sense together.

Significant (4 points)

Context
No context is needed. The joke $\begin{aligned} & \text { Some context is required. The joke } \\ & \text { Is there any context is logical and may reflect on may be somewhat unusual or re- } \\ & \text { necessary to under- mundane experiences. }\end{aligned}$

Context is required/not provided. The joke is unique and reflects on specific experiences or ones not commonly discussed. a lack of meaningful context?

Figure 2. Meme B1 (Score of 10)

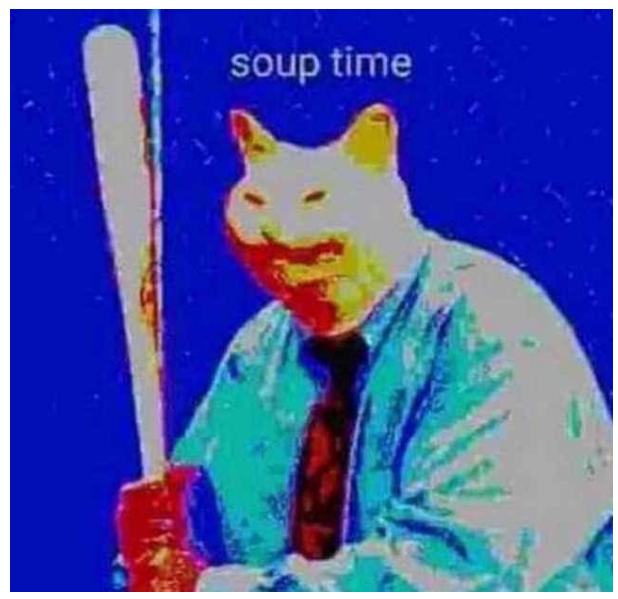

Chi-squared testing for difference was performed to analyze a select set of six memes from both surveys, representing a range of absurdity scores (Appendix $\mathrm{H}$ ). This testing was performed by the calculators available on the Social Science Statistics website. Chi-squared testing was selected as the most accurate method of analyzing the ordinal data collected from the two surveys to compare the Likert scale distributions. This testing revealed that a difference does exist, and we can be over $99 \%$ confident that absurdist humor content does affect the Likert scale humor rankings for this sample of Generation $\mathrm{Z}$ students. This relationship may not apply perfectly to all of Generation $\mathrm{Z}$, however; participants of this study were sampled from a predominantly white and upper-middle class neighborhood in the United States. Future research could analyze this relationship for a sample more indicative of Generation Z both within and outside of the United States, with a larger, more diverse group of participants.

\section{Conclusion}

From the data collected, a relationship between absurdist humor in memes and Generation $\mathrm{Z}$ humor rating has been highlighted. Absurdist humor does, in fact, influence humor rankings for Generation $\mathrm{Z}$ students as compared to less absurd content. This study further sought to find a way to codify absurdist humor while remaining impartial to the content of the meme sample provided to participants. The rubric created to quantify absurdity in visual stills has the 
potential to be adapted for video and audio meme formats following similar guidelines that reflect the components of each respective media type. This could potentially be replicated for other forms of humor to identify a humor style consistently and create a standard in the field of study for codifying humor types. Although the rubric only begins to define absurdity in visual elements, it could be refined through studies of specific humor types that present in any format.

The findings of this study bear implications for the existing body of knowledge as well. As previously noted, while accurate and cautious in analysis, the existing body of research has focused their analysis on outdated meme formats ("formats" referring to trends in memes including reused screencaptures or jokes being applied to different contexts) which could influences their perception of the online climate and how people interact with memes. While lag in the meme formats being analyzed is acceptable to some degree due to the rapidly changing tides of the internet and drastically different formats utilized by different social spheres, focusing analysis on memes relevant to the concerned population could create more accurate conclusions. We can see a link between one humor type and how one generation interacts with corresponding memes; future research could seek similar relationships between different formats and humor styles and different populations.

There are some limitations to the presented implications. Once again, the sample was not fully representative of the population, consisting of Generation $\mathrm{Z}$ as a whole. While this sample provides excellent insight into the relationship between absurd humor and meme humor perceptions, these findings could be best applied to the population after further research more indicative of the generation. Focusing on this sample, however, was an efficient way of avoiding confounding variables in meme enjoyment such as cultural background or socioeconomic status which can be better controlled for in a single high school.

A further use for these findings comes in the field of marketing and public relations. As marketing teams,- spanning the gamut from television ads to Instagram posts to Tweets mimicking a typical Twitter user-use memes, understanding humor preferences in a demographic could be greatly beneficial to marketing and social outreach. Knowing and understanding the background of a demographic is critical to effective outreach and growth and further tracking trends and humor styles could assist in that goal, especially for younger consumer groups. In a paper by librarian and social media consultant A. Woodworth, Woodworth notes the use of memes in the 2016 election cycle by Russian actors to influence voting and in the greater political conversation, reaffirming their presence in marketing and advertisement not just for products but for political ideology. Woodworth continues to suggest memes as a method of library outreach and following known meme formats. Understanding humor preferences in a demographic could once again make these methods more effective. Future studies could analyze the effectiveness of differing meme formats among demographics in certain marketing strategies or even the relationship between meme formats and relevance in political conversation.

Through the course of this study, meme humor was analyzed beyond simple jokes into something with more practical or even more sinister applications as has been utilized previously. When analyzing humor, things become infinitely less funny but make infinitely more sense as we further our understanding of this unique phenomenon. Something as simple as a cat's head on an apple can be quantified for humor types and rankings, but its social impact is an entirely different beast.

\section{Acknowledgements}

Thank you to my peers for consistent effort and support in the development of my paper and in the course of my research. And thank you to my parents, who have always supported my curiosity. 


\section{References}

Apter, E. (2019). Alphabetic Memes: Caricature, Satire, and Political Literacy in the Age of Trump. October, 170, 5-24.

Bourrier, K. (2016). Victorian Memes. Victorian Studies, 58(2), 272-282.

doi:10.2979/victorianstudies.58.2.08

Brown, A. (2020). Everything You\&\#39;ve Wanted to Know About Gen Z but Were Too Afraid to Ask. Forbes (September 23, 2020).

Cannizzaro, S. (2016). Internet memes as internet signs: A semiotic view of digital culture. Sign Systems Studies, 44(4), 562-586.

Dotterweich, J. (2019). An Argument for the Absurd. Live Ideas (February 2019), 50-57.

Glenwright, M., Tapley, B., Rano, J. K. S., \&amp; Pexman, P. M. (2017). Developing Appreciation for Sarcasm and Sarcastic Gossip: It Depends on Perspective. Journal of Speech, Language \&amp; Hearing Research, 60(11), 3295-3309.

Grundlingh, L. (2018). Memes as speech acts. Social Semiotics, 28(2), 147-168.

O’Meara, J. (2018). Meme Girls Versus Trump: Digitally Recycled Screen Dialogue as

Political Discourse. Velvet Light Trap: A Critical Journal of Film \&amp; Television, 82, $28-42$.

Sălcudean, M., \&amp; Motoroiu-Ştefan, R. (2020). Visual Humor through Internet Memes.

Iconicity, Irony, and Virality in the Digital Age (I). Revista Transilvania, 4, 67-72.

Snap Inc. More About Snap. (n.d.). https://www.newsroom.snap.com/about

Statistics Calculators. Quick Statistics Calculators. (n.d.). https://www.socscistatistics.com/tests/

TikTok. Our Mission. (n.d.). https://www.tiktok.com/about?lang=en

Turner, A. (2015). Generation Z: Technology and Social Interest. Journal of Individual Psychology, 71(2), 103-113.

van der Wal, A., Piotrowski, J. T., Fikkers, K. M., \&amp; Valkenburg, P. M. (2020). More than

Just a Laughing Matter: A Coding Framework of Humor in Media Entertainment for Tweens and Teens. Journal of Broadcasting \&amp; Electronic Media, 64(3), 478-498.

Woodworth, A. (2018). My Body is Ready: Best Practices for Using Memes on Library Social Media. Reference \&amp; User Services Quarterly, 58(2), 87-90. 